\title{
A Nursing Intelligence System to Support Secondary Use of Nursing Routine Data
}

W.O. Hackl'; F. Rauchegger²; E. Ammenwerth ${ }^{1}$

${ }^{1}$ Institute of Biomedical Informatics, UMIT-University of Health Sciences, Medical Informatics and Technology, Hall in Tirol, Austria; ${ }^{2}$ Nursing Management Board, Nursing Informatics, TILAK, Innsbruck, Austria

\section{Keywords}

Medical informatics, nursing informatics, databases, data integration, data mining

\section{Summary}

Background: Nursing care is facing exponential growth of information from nursing documentation. This amount of electronically available data collected routinely opens up new opportunities for secondary use.

Objectives: To present a case study of a nursing intelligence system for reusing routinely collected nursing documentation data for multiple purposes, including quality management of nursing care. Methods: The SPIRIT framework for systematically planning the reuse of clinical routine data was leveraged to design a nursing intelligence system which then was implemented using open source tools in a large university hospital group following the spiral model of software engineering. Results: The nursing intelligence system is in routine use now and updated regularly, and includes over 40 million data sets. It allows the outcome and quality analysis of data related to the nursing process.

Conclusions: Following a systematic approach for planning and designing a solution for reusing routine care data appeared to be successful. The resulting nursing intelligence system is useful in practice now, but remains malleable for future changes.

\section{Correspondence to:}

Dr. Werner 0. Hackl

Institute of Biomedical Informatics, UMIT-University of Health Sciences, Medical Informatics and Technology

Eduard Wallnöfer Zentrum 1

6060 Hall in Tirol, Austria

Email: werner.hackl@umit.at
Appl Clin Inform 2015; 6: 418-428

http://dx.doi.org/10.4338/ACI-2015-04-RA-0037

received: April 1, 2015

accepted in revised form: May 2, 2015

published: June 24, 2015

Citation: Hackl WO, Rauchegger F, Ammenwerth E

A Nursing Intelligence System to Support Secondary Use of Nursing Routine Data. Appl Clin Inform 2015; 6: 418-428 http://dx.doi.org/10.4338/ACl-

2015-04-RA-0037 


\section{Introduction}

The amount of electronically available clinical data in health care organizations is constantly growing [1]. Clinical data is documented to support the coordination and organization of patient care, to allow patient administration and billing, to support training, or for research purposes [2]. This increasing amount of available clinical data and information, together with new technologies and methodologies for the analysis of big data sets, open up new opportunities for secondary use of clinical data [3].

Secondary use means the use of clinical routine data outside direct care, including but not limited to analysis, research, quality measurement, safety measurement, or public health [4]. Secondary use comprises aggregating and comparing available data across time or populations to detect new and unexpected relationships in these data.

The increasing IT-based availability of structured clinical data also facilitates the definition and usage of clinical indicators. Quality indicators are increasingly important for health care and may help to measure quality of care, process quality, or patient satisfaction. A well designed indicator allows, in a standardized and quantifiable way, monitoring of the quality of a process or organization [5]. In recent years, the use of indicators to assess outcome quality and process quality has gained in importance in nursing care which is characterized by patient-centered care, inter-professional work, and increasing emphasis on quality and scientific evidence [6].

As in other fields within the health care industry, nursing care is facing an exponential growth of electronically available information from documentation. The existence of such an enormous mass of routinely generated health data opens up new possibilities for secondary use. However, building and analyzing dedicated integrated clinical and routinely collected nursing data repositories are nontrivial tasks due to methodological, ethical, legal, practical, and technical issues [4, 7]. Accordingly, most analysis functions within present nursing documentation systems are patient-centered and do not allow population-based analysis or indicator or quality measure development.

The objective of this paper is to present a case study of a nursing intelligence system. This nursing intelligence system is based on the secondary use of nursing data in order to develop and present quality indicators related to the nursing process and to determine the quality of nursing care. The overall aim is to verify that it is feasible to use routinely collected nursing data to define, integrate, and present nursing-related data to be used for nursing management and quality management.

\section{Methods}

Our approach is based on the SPIRIT methodology as a general framework for secondary use of clinical data. SPIRIT provides a general conceptual framework to systematically plan and design solutions for secondary use of clinical routine data. SPIRIT aims at tailoring such solutions to the specific needs of a single department without losing sight of the whole institution's needs. Results of this case study were also used to further refine SPIRIT. In the following, only a short overview is given. More details concerning the SPIRIT methodology can be found in $[8,9]$.

\subsection{The SPIRIT framework}

SPIRIT consists of three main blocks that are executed sequentially: i) Vision \& Strategy, ii) Analysis \& Specification, and iii) Feasibility \& Implementation.

The first block, "Vision \& Strategy", defines a vision and the main goals of the intended routine data reuse on a strategic level. In particular, a holistic perspective on the intended routine data reuse is crucial because different stakeholders and future users of a clinical routine data reuse solution in different organizational units may have different needs and requirements and may pursue different individual goals. Routine data reuse solutions usually evolve successively; single departments often pioneer such developments and intuitively implement solutions intended for specific purposes. Without anticipatory systematic planning of routine data reuse solutions, there is a risk of overfitting it to the boundary conditions of a single case. Therefore, it is crucial to align different possible individual goals strategically and to pursue a system-wide applicable concept. 
It is obvious that the success of any routine data reuse solution significantly depends on the perceived usefulness of information derived from it. Therefore, it is essential to know the specific questions and information needs of the different stakeholders as well as the available data sources and specific data elements. Thus, the second block, "Analysis \& Specification", is dedicated to an in-depth analysis of requirements from a future user's perspective (TOP-DOWN) and from a systems and data level (BOTTOM-UP). A comprehensive analysis of the clinical context in which the data is produced has to be conducted to complement these two contrary analysis tracks (IN-BETWEEN).

The third block, "Feasibility \& Implementation", is dedicated to the design and implementation of solutions of data integration and data management (Clinical Data Marts and Warehouses) as well as data analysis (Clinical Intelligence Systems). Comprehensive feasibility studies precede the design phases and deliver essential information on framework conditions for the design and the subsequent implementation phases.

\subsection{The case study}

We chose a single case design, selecting as setting the Tyrol's hospital group (Tiroler Landeskrankenanstalten $\mathrm{GmbH}$ - TILAK), which comprises the University Hospitals of Innsbruck plus several district and specialty hospitals. The setting was chosen for the following reasons: In 2006 an electronic nursing documentation system was implemented at the University Hospitals of Innsbruck, with a large amount of nursing data from inpatient and outpatient areas available in electronic form. Since then, the electronic nursing documentation system has been rolled out in almost all of the group's hospitals. Documentation includes all phases of the nursing process and is highly structured. Standardized nursing guidelines for all patient stays are available within the documentation system. NANDA-I (NANDA International, formerly the North American Nursing Diagnosis Association) is used to classify nursing diagnosis. For the coding of nursing interventions and nursing outcomes, internal proprietary catalogs are used. For different assessments, standard instruments (e.g. BarthelIndex [10], Braden Scale [11]) as well as proprietary tools (e.g. initial nursing assessment, fall investigation, decubitus inquiry) are used.

Nursing management had a strong interest in analyzing the available routine data in order to optimize the nursing documentation system and to allow the development of population-based indicators for care process and outcome quality. All this made TILAK a good setting for a case study of a nursing intelligence system.

The hospitals of the TILAK group have a total of 2,465 beds. The mean duration of stay at the University Hospitals of Innsbruck is 4.8 days. TILAK employs 6,400 full-time equivalent (FTE) health care professionals (among them 2,470 FTE registered nurses) who handle 120,000 inpatient (760,000 care days) and 1.15 million outpatient visits per year (2013 data). The architecture of the TILAK hospital information system is characterized by over 150 applications with a few "leading applications", including a patient administration system and a clinical information system. Nursing documentation is part of this clinical information system and covers all aspects of the nursing process [12].

\subsection{Implementation}

After defining a nursing data reuse vision and strategy, a thorough system analysis was conducted that focused on available documentation systems and their data elements (BOTTOM-UP). Nursing documentation processes were observed and nurses were interviewed (IN-BETWEEN), and nursing managers were interviewed regarding needed functionality for a nursing intelligence system (TOPDOWN). A list of requested analysis questions was developed and compared with the available routine data elements.

Then requirements regarding data models, data flows, reporting, data security, and data safety were aggregated. To test the feasibility of the approach, a first prototype based on a data warehouse reference model [13] was developed and its functionality verified in several workshops with nurse management. Test runs with anonymized test data helped to verify technical and performance issues. 
The software engineering process was based on the spiral model by Boehm [14]. Freely available open source tools for data warehousing and business intelligence were used for the implementation. For data extraction, export functions of the clinical documentation system were used. For data integration and the ETL process (extraction - transform - load), Talend Open Studio for data integration (www.talend.com) was used. For data management, a PostgreSQL (www.postgresql.org) database was used. For data analysis, business intelligence and result presentation, BIRT (Business Intelligence and Reporting Toolkit, www.eclipse.org/birt) was used.

\section{Results}

The final nursing intelligence system comprises two tiers: A data integration and management layer providing a nursing data mart; and a data analysis layer providing a nursing intelligence and reporting platform with predefined customizable reports. The reports can be accessed via a web-based user interface. In addition, ad-hoc queries, filtering, and OLAP functionalities (online analytical processing, e.g. drill-in, drill-through, roll-up) were made available over the web interface. Analysis results are presented within a browser window and can be saved in different file formats (e.g. PDF, DOC, XLS). It is also possible to export the raw or processed data into .csv format for further analysis outside the web-based platform. For advanced analysis, individual queries and data exports from the nursing data mart are available ( $>$ Figure 1$)$.

The analysis questions were systematically derived and addressed quality of documentation (e.g. completeness of documentation), process indicators for nursing documentation (e.g. time for documentation), indicators for nursing care processes (e.g. statistics on nursing diagnosis, goals and interventions), and indicators for quality of nursing care (e.g. statistics on nursing evaluation, interval between admission and start of a nursing diagnosis, interval between start of a nursing diagnosis and its first evaluation). Overall, over 50 different analysis questions were defined. $>$ Figure 2 gives an overview of the categories of questions, the basic selection and filter criteria, and the stratification parameters.

Based on the analysis questions and the available data elements, a data model was established as basis for the nursing intelligence system ( $>$ Figure 3). The data model contains two central elements: the relation "patient", holding a unique patient ID for each patient, and the relation „stay", holding a unique ID for every visit/admission. These two IDs are valid in the whole hospital group and every data element from any documentation system within TILAK can be integrated into the data model and linked with other elements using these IDs. In the existing data model, some relations (e.g. the nursing assessment) are directly linked to both central elements whereas others are only linked to one element (e.g. nursing inspections or nursing diagnoses have no direct link to the patient as they depend on the respective stays). Redundancies within the data model are intended; due to performance issues, normalization was not applied.

Until March 2015, data from 190,000 patients and 445,000 admissions had been extracted and imported into the nursing intelligence system. Around 8,000 new admissions are imported each month ( $>$ Figure 4). The nursing intelligence system has been in routine use since 11/2009 and is being used as a management tool by the TILAK nursing management board.

\section{Discussion}

Due to the rising amount of available electronic documentation, secondary data use has gained in importance in recent years. This also holds true for nursing care. Using an available framework for secondary use of clinical data (SPIRIT), a nursing intelligence system was developed and implemented at the University Hospital of Innsbruck.

To our knowledge, this is one of the first publications introducing a systematically planned and successfully implemented system for secondary use of "big" routinely collected data in nursing care. Other approaches (e.g. [15]) have focused on pilots and concepts, but did not lead to comprehensive and adaptable nursing intelligence systems for routine use. 
In the case study, we learned that building a nursing intelligence system demands not only methodologies for data warehousing and secondary data use, but also in-depth knowledge of the nursing processes and underlying data models as well as of the availability of structured nursing documentation data. The insights gained during this case study led to further refinement of the SPIRIT framework.

The SPIRIT framework is built on available frameworks for data warehouses and secondary data use (e.g. [16], [17]). As in other frameworks, organizational and technical issues play an important role, but SPIRIT additionally pays particular attention to the specific conditions of the clinical domain. In-depth and systematic analysis of clinical documentation systems and available data elements, definition of analysis questions, analysis of the clinical context and the clinical processes in which these data elements are produced, and analysis of how the resulting information will be used are necessary. SPIRIT also helps to keep a holistic perspective by starting with the definition of a data reuse vision and strategy.

This approach was found quite helpful, as it fostered discussion among the nursing managers on the issues they are really interested in. Many of the identified analysis questions have already been implemented in the nursing intelligence system. For implementation, only open source tools were used. Due to the modular concept, all components can be substituted by commercial products if advanced functionalities, which are not available within open source tools, are needed.

In the context of secondary use of health data, the term "gold mine" or "diamond mine" is sometimes used $[18,19]$. However, as our experience shows, it is relatively easy to analyze routine data to answer simple questions such as "how many, how long, how often..." But to identify cross-data relationships, more complex analyses, such as different machine learning techniques or methods from complex network science [20], are needed.

Here, an intelligence system that allows easy-to-use and flexible ad-hoc analysis of the data can be a powerful tool for stakeholders such as nursing managers. These flexible and ad-hoc analyses are possible in our nursing intelligence system. For more advanced analyses, individual queries of the nursing data mart and separate analyses outside the standard analysis platform can be performed.

In addition, the intelligence system supports the integration of new data sources. This is absolutely necessary in a rapidly changing health care context where new evidence, new clinical and nursing pathways, new analysis questions, and new data sources as well as new data elements continue to emerge. As an example, questions related to quality and quantity of nursing interventions were not implemented in the initial case study, but related data and analysis reports are now integrated in the running nursing intelligence system.

\section{Conclusion}

We developed a nursing intelligence system in a large university hospital with a high availability of structured electronic nursing documentation as case study. Since rollout other health care organizations from the same hospital group have also started to use the nursing intelligence system. Building a nursing intelligence system is naturally dependent on the availability of structured information, even when efforts to analyze free text from clinical documentation to support populationbased analysis have been launched in recent years [21].

The nursing intelligence system is now in routine use and is updated regularly using semi-automatic routines. Nursing managers are able to use the nursing intelligence system to execute predefined queries or ad-hoc queries. The system is adaptable and extendible with little effort to respond to new and emerging fields of interest.

\section{Clinical Relevance}

Our experiences are generalizable to other health care organizations with a comparable level of electronic documentation. A systematic approach for planning and designing a solution for reusing routine care data was successful. The resulting nursing intelligence system is applicable in practice now, but remains malleable for future changes. 


\section{Conflict of Interest}

The authors declare that they have no conflicts of interest in the research.

\section{Human Subjects Protections}

The procedures used have been reviewed in compliance with ethical standards of the responsible committee on human experimentation.

\section{Acknowledgments}

We would like to thank our students in the biomedical informatics bachelor programs who contributed to the case study. We would also like to thank the Tyrolean federal hospitals group, TILAK (nursing management board / nursing informatics), for the possibility to conduct the case study and the TILAK information technology department for partial funding. Parts of this work were also funded by Tiroler Wissenschaftsfonds TWF (UNI-0404-1014, 0404-1189). 


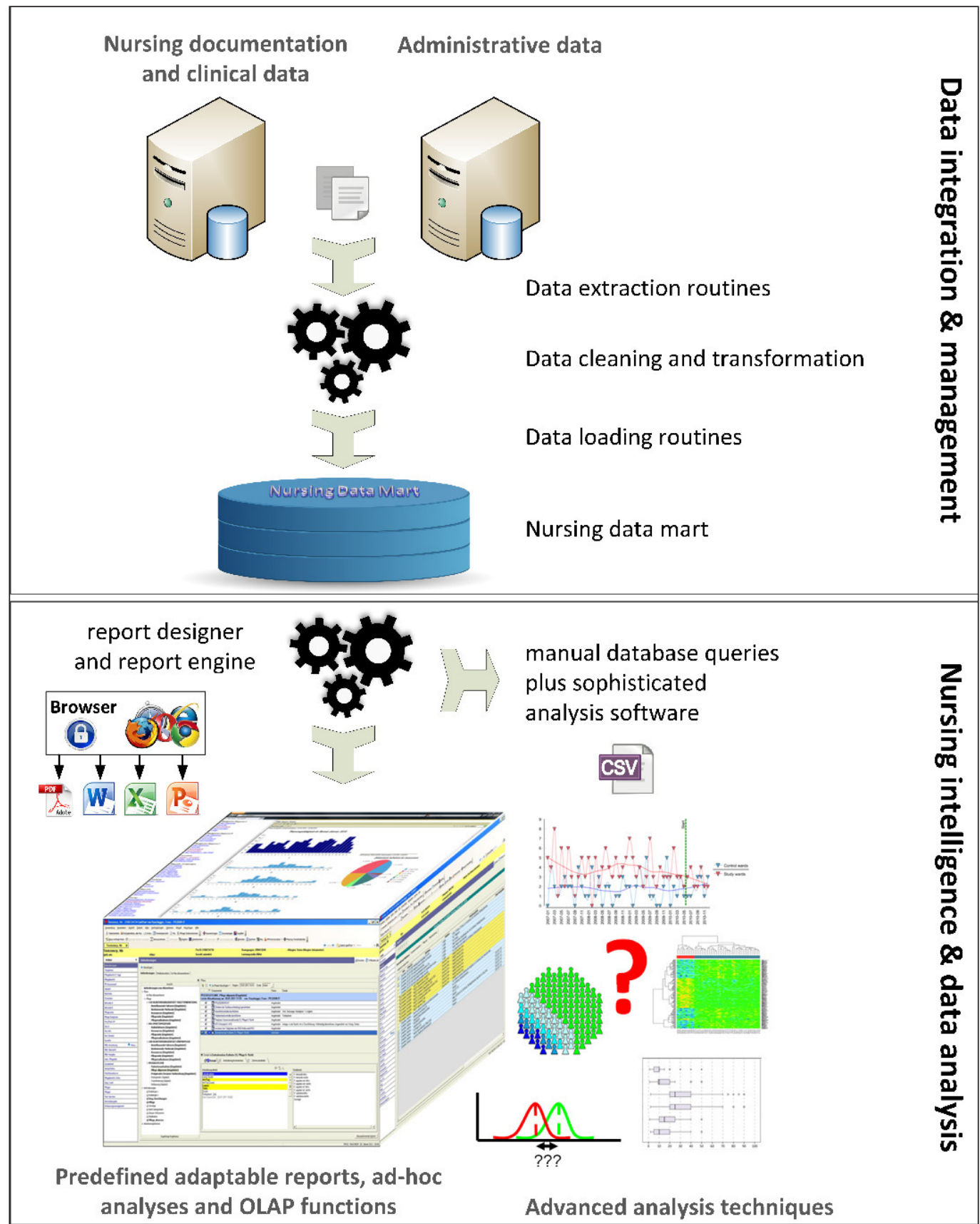

Fig. 1 Overview of the architecture of the nursing intelligence system consisting of two layers. The first layer is dedicated to data integration and management and holds the data extraction, transformation, and loading routines as well as the integrated data repository, a so-called nursing data mart. The second layer, dedicated to data analysis, provides a web-based analysis platform with predefined, adaptable reports for standard analyses. For special analyses and advanced analysis techniques, separate subsets from the nursing data mart can be obtained by individual queries. 


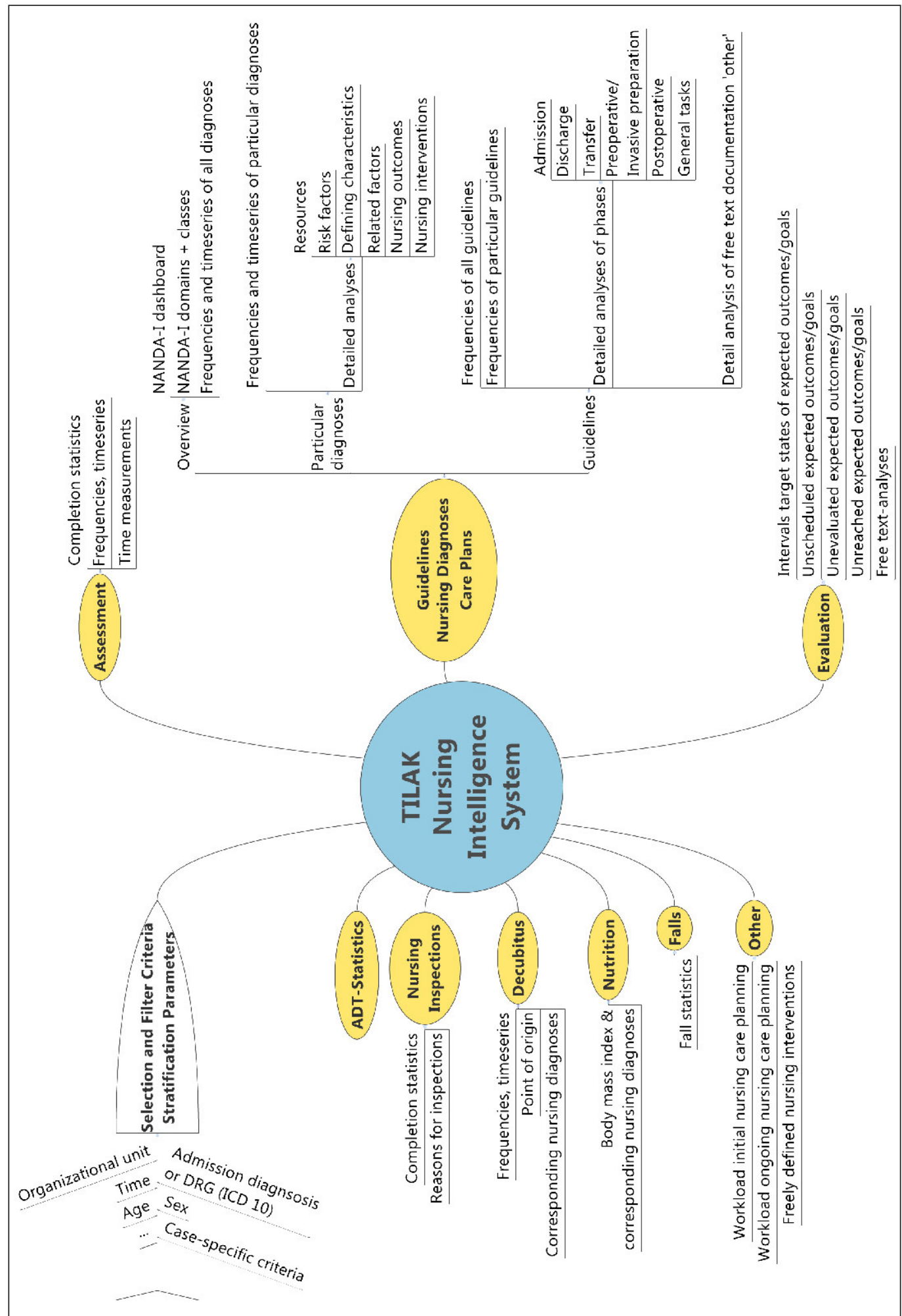

Fig. 2 Overview of the groups of different analysis questions, of the selection and filter criteria and of the stratification parameters available in the nursing intelligence system. 


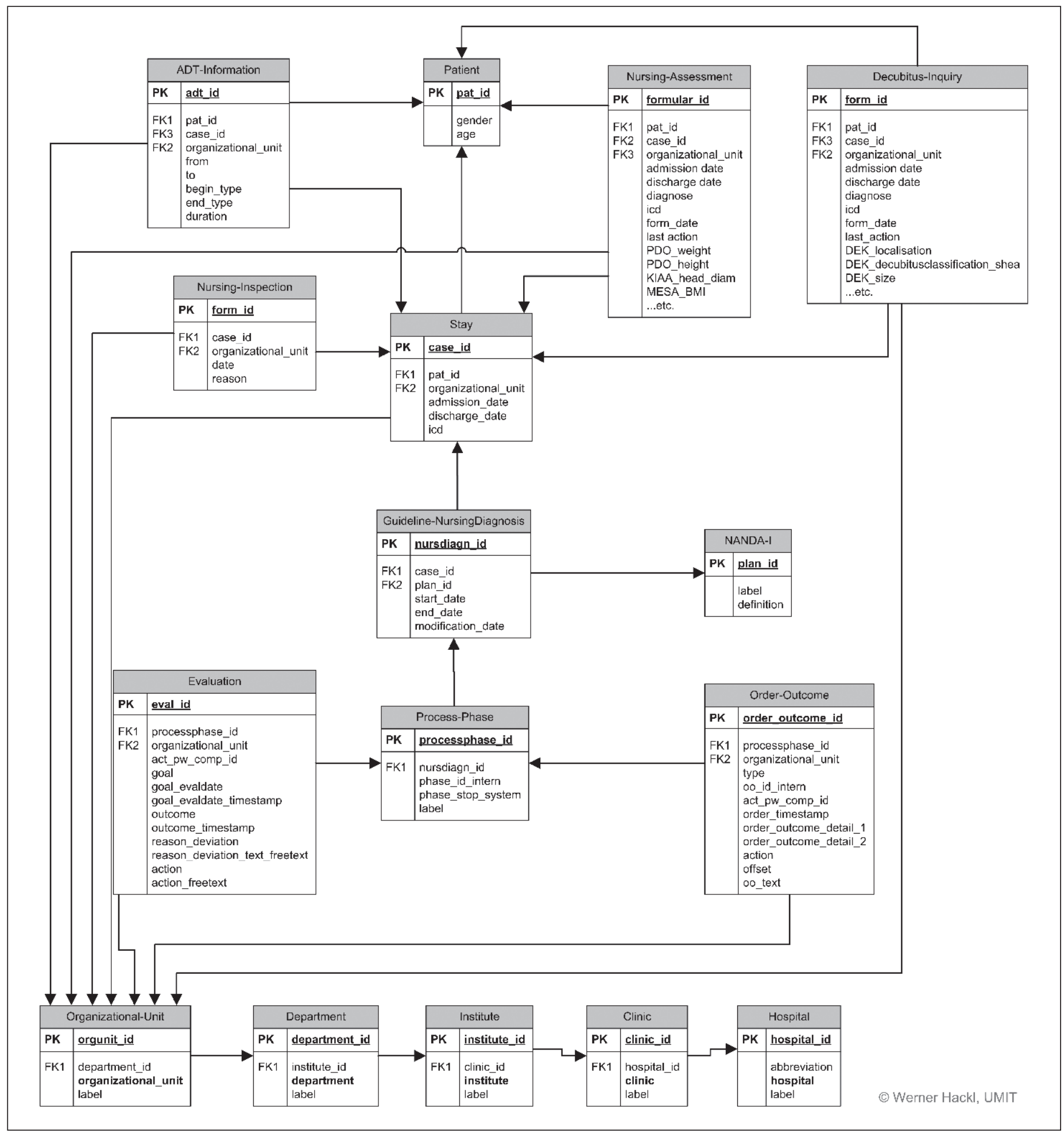

Fig. 3 Data model of the nursing intelligence system with the relations "Patient" and "Stay". Redundancies within the data model are intended; due to performance issues, normalization was not applied (only major tables and attributes are shown). 


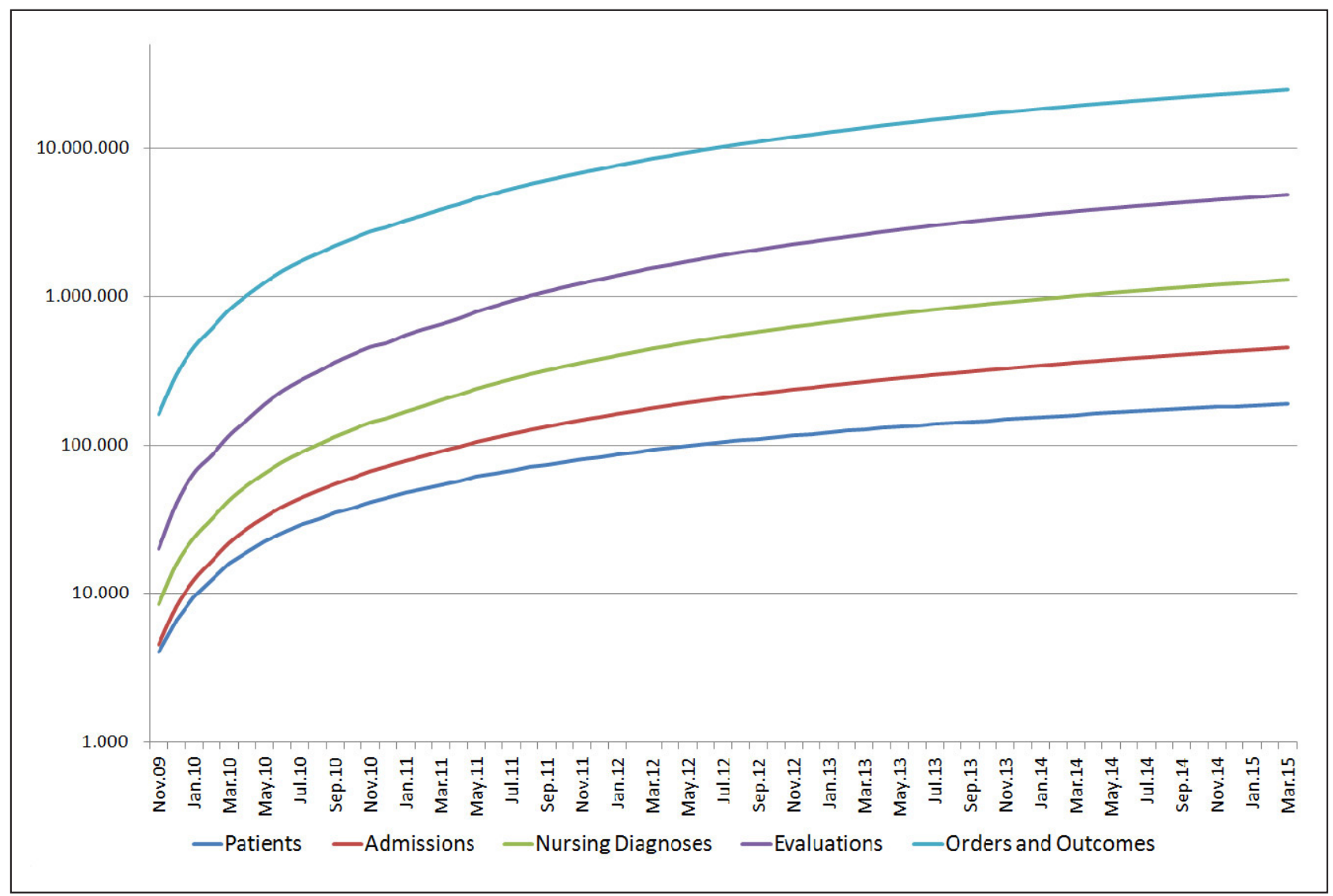

Fig. 4 Development of the amount of data (physical patients, admissions, nursing diagnoses including pathways, evaluations of nursing goals, orders and outcomes) in the nursing intelligence system since 11/2009 (logarithmic scale). 


\section{References}

1. Berner E, Moss J. Informatics Challenges for the Impending Patient Information Explosion. J Am Med Inform Assoc 2005; 12(6): 614-617.

2. Leiner F, Haux R. Systematic Planning of Clinical Documentation. Methods Inf Med 1996; 35(1): 25-34.

3. Mayer-Schönberger V, Cukier K. Big Data: A Revolution that Will Transform how We Live, Work, and Think: Houghton Mifflin Harcourt; 2013.

4. Safran C, Bloomrosen M, Hammond WE, Labkoff S, Markel-Fox S, Tang PC, Detmer DE, Expert P. Toward a national framework for the secondary use of health data: an American Medical Informatics Association White Paper. J Am Med Inform Assoc 2007; 14(1): 1-9.

5. Joint Commission on Accreditation of Healthcare Organizations. Primer on indicator development and application : measuring quality in health care. Oakbrook Terrace, Ill.: Joint Commission on Accreditation of Healthcare Organizations; 1990.

6. Burston S, Chaboyer W, Gillespie B. Nurse-sensitive indicators suitable to reflect nursing care quality: a review and discussion of issues. J Clin Nurs 2014; 23(13-14): 1785-1795.

7. Prokosch HU, Ganslandt T. Perspectives for medical informatics. Reusing the electronic medical record for clinical research. Methods Inf Med 2009; 48(1): 38-44.

8. Hackl WO. Erschließung und Sekundärnutzung von Routinedaten aus der klinischen und pflegerischen Prozessdokumentation: Ein Rahmenkonzept, Vorgehensmodell und Leitfaden. Hall in Tirol: UMIT - University for Health Sciences, Medical Informatics and Technology; 2013.

9. Hackl WO, Ammenwerth E. SPIRIT: A Conceptual Framework and Procedure Model for Systematic Planning of Intelligent Reuse of Integrated Clinical Routine Data. Methods Inf Med. 2015;submitted

10. Mahoney FI, Barthel DW. Functional Evaluation: The Barthel Index. Md State Med J 1965; 14: 61-65.

11. Braden BJ, Bergstrom N. Clinical utility of the Braden scale for Predicting Pressure Sore Risk. Decubitus 1989; 2(3): 44-46, 50-51.

12. Ammenwerth E, Buchberger W, Kofler A, Krismer M, Lechleitner G, Pfeiffer K-P, Schaubmayr C, Triendl C, Vogl R. IT-Strategie 2008 - 2012 der Tiroler Landeskrankenanstalten (TILAK): Informationstechnologie im Dienste von Medizin und Pflege 2007.

13. Bauer A, Günzel H. Data-Warehouse-Systeme: Architektur, Entwicklung, Anwendung. Heidelberg: dpunkt-Verlag; 2009.

14. Boehm B. A spiral model of software development and enhancement. IEEE Computer. 1988; 21(5): 61-72.

15.Junttila K, Meretoja R, Seppala A, Tolppanen EM, Ala-Nikkola T, Silvennoinen L. Data warehouse approach to nursing management. J Nurs Manag 2007; 15(2): 155-161.

16.Li P, Wu T, Chen M, Zhou B, Xu WG. A study on building data warehouse of hospital information system. Chin Med J (Engl) 2011; 124(15): 2372-2377.

17. Samaha T, Croll P. A Data Warehouse Architecture for Clinical Data Warehousing. In: Roddick F, Warren J, editors. Proceedings Australasian Workshop on Health Knowledge Management and Discovery (HKMD 2007) CRPIT; Ballarat, Victoria: Australian Computer Society 2007: 227-232.

18. Aller RD. The clinical laboratory data warehouse. An overlooked diamond mine. Am J Clin Pathol 2003; 120(6): 817-819.

19. Webster PC. Sweden's health data goldmine. CMAJ. 2014; 186(9): E310.

20.Dehmer M, Hackl WO, Emmert-Streib F, Schulc E, Them C. Network nursing: connections between nursing and complex network science. Int J Nurs Knowl 2013; 24(3): 150-156.

21. Collins SA, Cato K, Albers D, Scott K, Stetson PD, Bakken S, Vawdrey DK. Relationship between nursing documentation and patients' mortality. Am J Crit Care 2013; 22(4): 306-313. 